\title{
The impact of additional epicardial imaging to transesophageal echocardiography on intraoperative detection of residual lesions in congenital heart surgery
}

\author{
Andreea Dragulescu, MD, PhD, ${ }^{\mathrm{a}}$ Fraser Golding, MD, ${ }^{\mathrm{a}}$ Glen Van Arsdell, MD, ${ }^{\mathrm{a}}$ \\ Christopher Caldarone, MD, ${ }^{\mathrm{a}}$ Luc Mertens, MD, PhD, ${ }^{\mathrm{a}}$ Osman Al-Radi, MD, ${ }^{\mathrm{b}, \mathrm{c}}$ and Kyong-Jin Lee, MD ${ }^{\mathrm{a}}$
}

\begin{abstract}
Objective: Transesophageal echocardiography is the primary intraoperative imaging modality used to evaluate cardiac surgery. Its predecessor, epicardial echocardiography, enables visualization of certain cardiovascular structures that are beyond the visual scope of transesophageal echocardiography. We review the current use of epicardial echocardiography to analyze its contemporary application and benefit.
\end{abstract}

\begin{abstract}
Methods: A retrospective review of the intraoperative echocardiograms of 1204 children undergoing bypass cardiovascular surgery between January 2007 and December 2009 was performed. The incidence of epicardial echocardiography use, intraoperative revisions, and early reinterventions were analyzed.

Results: Epicardial echocardiography was performed in $7.9 \%$ of all intraoperative studies: epicardial echocardiography alone $(n=38)$ and transesophageal echocardiography + epicardial echocardiography $(n=57)$. Epicardial echocardiography alone was performed in patients with contraindications for transesophageal echocardiography. In the transesophageal echocardiography + epicardial echocardiography group, indications to obtain additional information by epicardial echocardiography were for the assessment of branch pulmonary arteries $(40 \%)$, coronary arteries (28\%), aortic arch/Blalock-Taussig shunt (14\%), Glenn/Fontan circuit (9\%), pulmonary veins/baffles $(7 \%)$, and residual ventricular septal defects $(1.7 \%)$. The overall intraoperative surgical revision incidence was $10.2 \%$, consisting of $21 \%$ of the transesophageal echocardiography + epicardial echocardiography group, $5.3 \%$ of the epicardial echocardiography alone group $(P=.01)$, and $9.8 \%$ of transesophageal echocardiography alone group $(P=.02)$. Intraoperative revisions indicated after epicardial echocardiography were mostly related to extracardiac structures $(77 \%)$, whereas they were mostly related to intracardiac structures in the transesophageal echocardiography alone group $(80.7 \%)(P=.0002)$. Early reintervention was indicated mostly for pulmonary artery and Glenn obstructions, the majority $(75 \%)$ with previously known stenosis or interventions on the pulmonary arteries.
\end{abstract}

Conclusions: Epicardial echocardiography detects residual intraoperative lesions not visualized by transesophageal echocardiography, most frequently related to pulmonary arteries. Its use, in addition to standard transesophageal echocardiography, may decrease the need for early reintervention. (J Thorac Cardiovasc Surg 2012;143:361-7)

\section{Supplemental material is available online.}

Residual cardiovascular lesions, anatomic or functional, ${ }^{1,2}$ are a major cause of morbidity and mortality after

\footnotetext{
From the Divisions of Cardiology and Cardiovascular Surgery, ${ }^{\mathrm{b}}$ The Labatt Family Heart Centre, Department of Paediatrics, Hospital for Sick Children and University of Toronto, Toronto, Ontario, Canada; and Department of Surgery, ${ }^{\mathrm{c}}$ King Abdulaziz University, Jeddah, Saudi Arabia.

Disclosures: Authors have nothing to disclose with regard to commercial support.

Received for publication Feb 8, 2011; revisions received May 9, 2011; accepted for publication June 7, 2011; available ahead of print July 13, 2011.

Address for reprints: Kyong-Jin Lee, MD, The Labatt Family Heart Centre, The Hospital for Sick Children, 555, University Avenue, Toronto, M5G 1X8, Canada (E-mail: kyong-jin.lee@sickkids.ca).

0022-5223/\$36.00

Crown Copyright (C) 2012 Published by Elsevier Inc. on behalf of The American Association for Thoracic Surgery

doi:10.1016/j.jtcvs.2011.06.010
}

congenital heart surgery. The introduction of intraoperative epicardial echocardiography (EpE) more than 3 decades ago allowed for prompt recognition of significant defects and affected the rate of immediate surgical revision. $^{3-6}$ Most commonly, reoperation involved atrioventricular and aortic valves and outflow tract reconstructions. $^{7,8}$ In the early 1990s, transesophageal echocardiography (TEE) was validated against EpE and has largely replaced it, ${ }^{9,10}$ although it has remained as a back-up imaging modality in cases in which TEE is contraindicated or the probe cannot be inserted. There are, however, limitations in TEE imaging, particularly for branch pulmonary arteries, aortic arch, coronary arteries, and segments of the superior vena cava. ${ }^{11}$ In the modern era of complex congenital heart surgery, visualization of these structures is more prudent. This study reviews the current use of $\mathrm{EpE}$ in the detection of postoperative residual lesions. 


\section{Abbreviations and Acronyms \\ EpE $=$ epicardial echocardiography \\ TEE $=$ transesophageal echocardiography}

\section{MATERIALS AND METHODS \\ Patient Population}

All pediatric patients with intraoperative echocardiography undergoing open surgery between January 2007 and December 2009 were retrospectively identified. At The Hospital for Sick Children, all cardiopulmonary bypass operations, with the exception of secundum atrial septal defect repairs, include intraoperative echocardiographic imaging. The Heart Centre database and patient health records were reviewed. The study was approved by the hospital research ethics board. Data collection included cardiac diagnoses and surgical procedure, type and findings of intraoperative echocardiographic imaging, indications for epicardial imaging, type of surgical revision, and echocardiographic "misses," defined as lesions not identified by intraoperative echocardiography that required reintervention within the first postoperative year.

Intraoperative echocardiographic studies were performed with the commercially available Philips IE-33 echocardiographic system (Philips Healthcare, Andover, Mass), using the S7-3t pediatric TEE probe in patients less than $15 \mathrm{~kg}$, the $\mathrm{S} 7-2$ Omni TEE probe in patients more than $15 \mathrm{~kg}$, and the X7-2t 3D TEE probe in patients more than $20 \mathrm{~kg}$. For epicardial imaging the S12-4 sector array probe was used. All intraoperative echocardiograms and written reports were reviewed.

We compared 3 groups: patients undergoing TEE alone, patients undergoing EpE alone, and patients undergoing EpE in addition to TEE (TEE + EpE). All studies were reviewed with regard to the cardiac structures imaged and the pressure gradients obtained. In the cases in which EpE was obtained in addition to TEE, the additional cardiac structures imaged were noted, and unless specifically stated, the assumption was made that EpE was performed to obtain imaging of these particular structures.

\section{Statistical Analysis}

Descriptive statistics were used to characterize the study population. Values were expressed as median and range for continuous variables. Logistic regression models were used to assess correlations between echocardiographic findings and patient characteristics. Pearson's chi-square test and Fisher exact test were used to compare different subgroups. All data analysis was performed with SPSS 15.0 for Windows (SPSS Inc, Chicago, Ill).

\section{RESULTS}

Data from 1204 surgical interventions with intraoperative echocardiograms were analyzed. Fifty-four percent of the patient population was male. Cardiac surgery was performed at a median age of 0.7 years (range $0-18.4$ years) and a median weight of $7.5 \mathrm{~kg}$ (range $1.9-140 \mathrm{~kg}$ ). No major complications related to the use of TEE or EpE were noted from the health records. The diagnostic subgroups are presented in Table E1.

\section{Use of Epicardial Echocardiography}

EpE was performed alone or in conjunction with TEE in 95 patients $(7.9 \%)$. It was the only imaging modality in 29 patients, related to failure to insert the TEE probe in 18 patients, contraindications for TEE use in 6 patients, and for unknown reasons in 5 patients. Hemodynamic instability or airway obstruction caused by the TEE probe was documented in 9 patients $(9.5 \%)$, and further imaging was obtained by EpE. Because TEE imaging, if present, was extremely limited, we included these cases in the EpE alone group for the analysis (total $\mathrm{n}=38$ for EpE alone group). For the TEE + EpE group $(n=57)$, the most common indications for obtaining additional imaging by EpE were for the branch pulmonary arteries $(40.3 \%)$ (Figure 1$)$, coronary arteries $(28.1 \%)$ (Figure 2), and aortic arch (14\%). Other indications were Glenn/Fontan circuits $(8.8 \%)$, pulmonary veins/venous baffle $(7 \%)$, and residual ventricular septal defect $(1.7 \%)$. During the study period, there was a significant increase in the use of $\operatorname{EpE}(P<.01)$ to supplement TEE, particularly for imaging the pulmonary $(P=.002)$ and coronary arteries $(P=.035)$, whereas the incidence of $\mathrm{EpE}$ alone remained similar $(P=.45)$ (Figure 3$)$.

\section{Intraoperative Surgical Revision}

Intraoperative surgical revision was performed in 10.2\% of the 1204 patients (Table 1). It was indicated in 14 patients $(14.7 \%)$ when EpE was used compared with $9.8 \%$ in the TEE only group $(P=.09)$. The TEE $+\mathrm{EpE}$ subgroup had the highest surgical revision incidence of $21.1 \%(P=.02)$. There was no increase in the intraoperative revision rate over time, but there was an increased trend in the EpE group: $6.4 \%$ in 2007 and $9 \%$ in 2008 and $17.2 \%$ in $2009(P=.06)$. Of the 14 patients who had EpE and surgical revision, in 13 it was the epicardial imaging that identified important residual lesions for reoperation. The majority $(77 \%)$ were related to extracardiac structures. In contrast, the main indication for surgical revision in the TEE only group was for intracardiac abnormalities $(80.7 \%)(P=.0002)$.

\section{Intraoperative Echocardiographic Misses in Patients Undergoing Early Reinterventions}

Intraoperative echocardiographic misses requiring reintervention within 1 year occurred in 29 patients $(2.4 \%)$ to address 38 missed residual lesions. Structures that were intraoperatively sufficiently imaged and accurately interpreted not to have significant abnormalities warranting intraoperative revision but later required reintervention were not included for further analysis. This was based on the assumption that these lesions represented natural postoperative evolution. Most often, the lesion involved the left $(42.1 \%)$ and right $(21 \%)$ pulmonary arteries, the Glenn anastomosis $(15.8 \%)$, and the coronary arteries $(7.9 \%)$ (Table 2). In 4 of the 29 patients, the residual lesion was imaged by TEE but not correctly identified on the echocardiographic report, and no intraoperative revision was performed. Of the 21 patients who required reintervention for pulmonary artery or Glenn obstruction, 15 had a preoperatively known stenosis or intervention. 

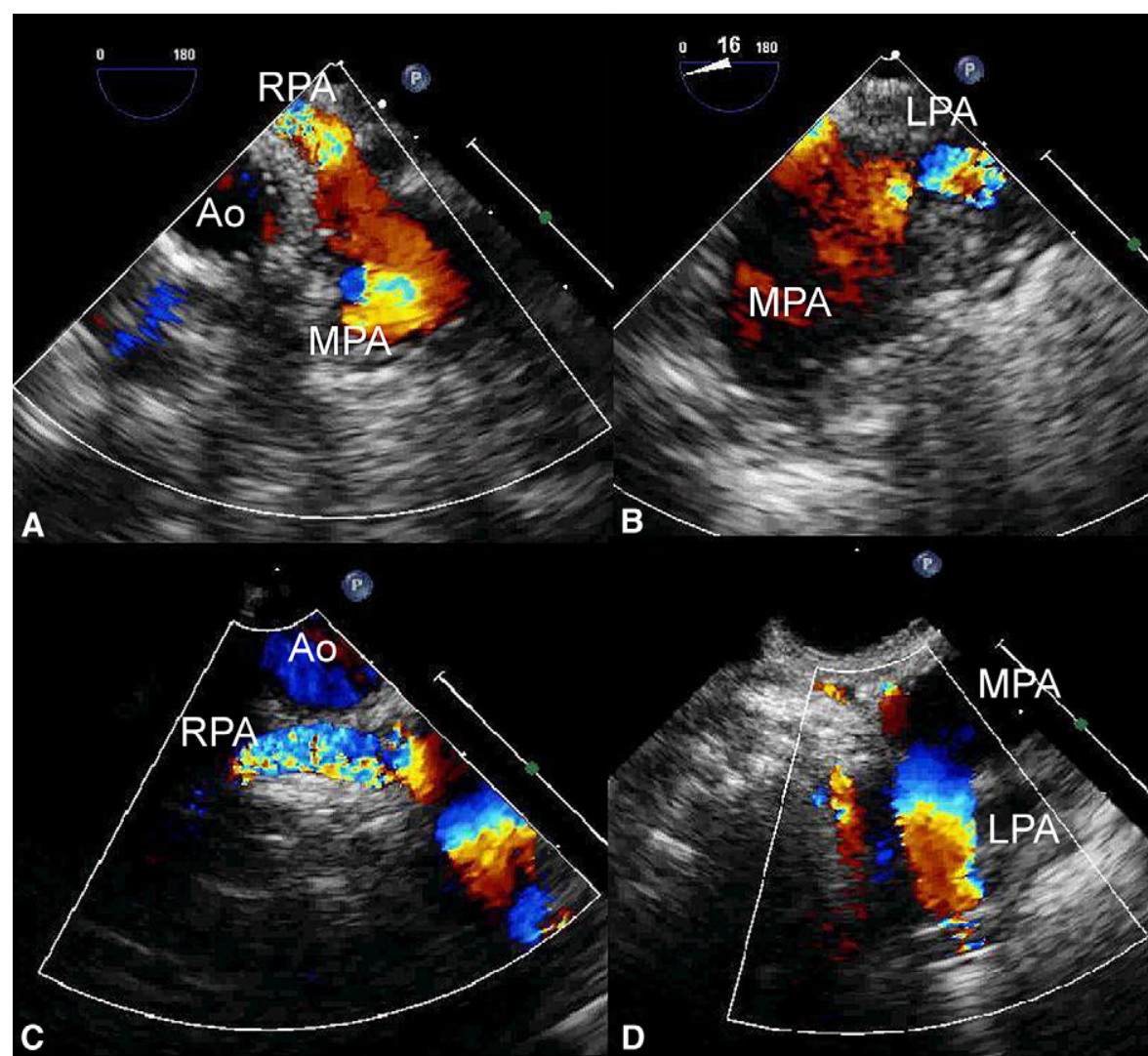

( )

(3)

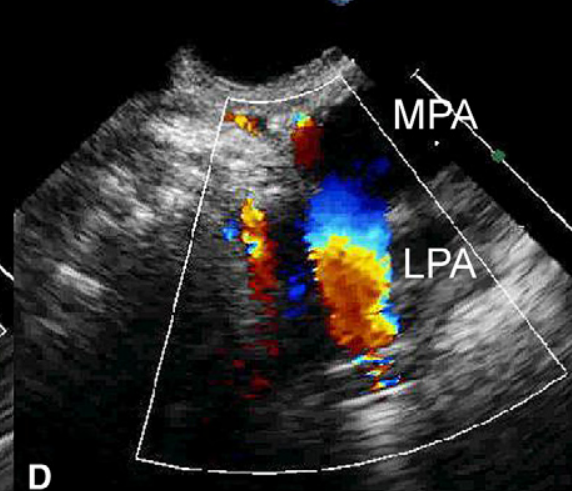

D

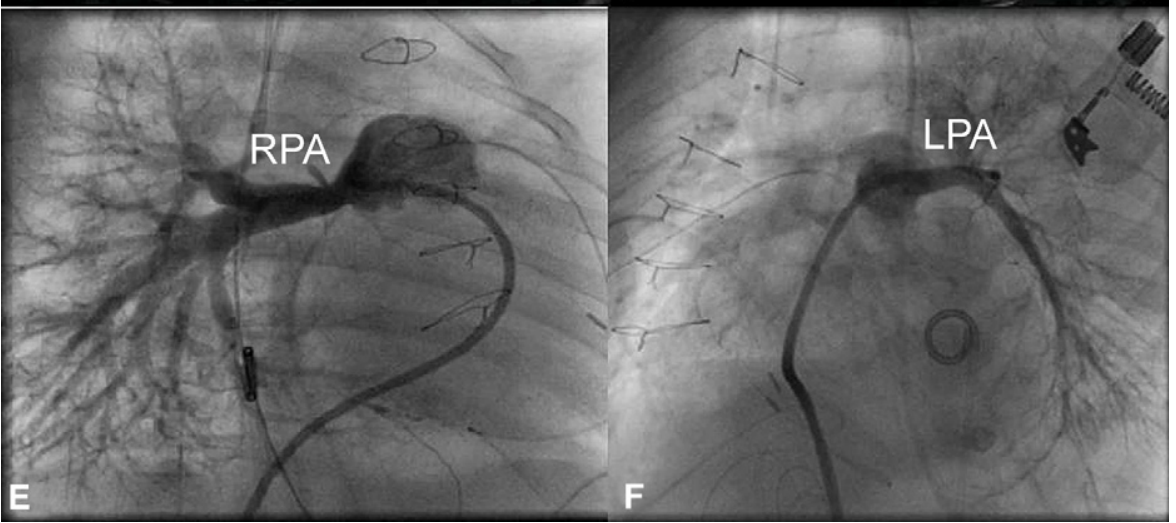

FIGURE 1. Intraoperative echocardiography in a 5-month-old child after repair of Fallot-type double outlet right ventricle. Postoperative TEE showed a good intracardiac result but turbulence at the origin of the pulmonary arteries (A, B). Further epicardial imaging confirmed narrowing at the origin of the right pulmonary artery (C) and small left pulmonary artery (D) with significant gradients. Diagnostic catheterization (E, F) showed suprasystemic right ventricular pressures, stenosis at the origin of right pulmonary artery (peak gradient $33 \mathrm{~mm} \mathrm{Hg}$ ), and diffusely hypoplastic left pulmonary artery. The patient underwent surgical revision for patch enlargement of branch pulmonary arteries with a good result. Ao, Aorta; $L P A$, left pulmonary artery; MPA, main pulmonary artery; $R P A$, right pulmonary artery.

In the TEE alone group, reinterventions were performed on the pulmonary arteries or Glenn anastomoses in 18 of 21 patients (Table 2). In $77.8 \%$ of patients, the primary cardiac diagnosis and surgery would have deemed these structures as being at risk for residual abnormalities. For example, during the study period there were 14 patients who underwent stage 2 hybrid procedure for hypoplastic left heart syndrome. In 3 of the first 11 patients, there were missed lesions by TEE requiring early reinterventions on the pulmonary arteries or Glenn anastomosis. For the last 3 patients, exit angiography was performed that demonstrated residual left pulmonary artery stenosis in 2 , and this was addressed intraoperatively.

Two patients in the EpE alone group had residual lesions that were not identified by intraoperative echocardiography but were of clinical significance in the postoperative period 


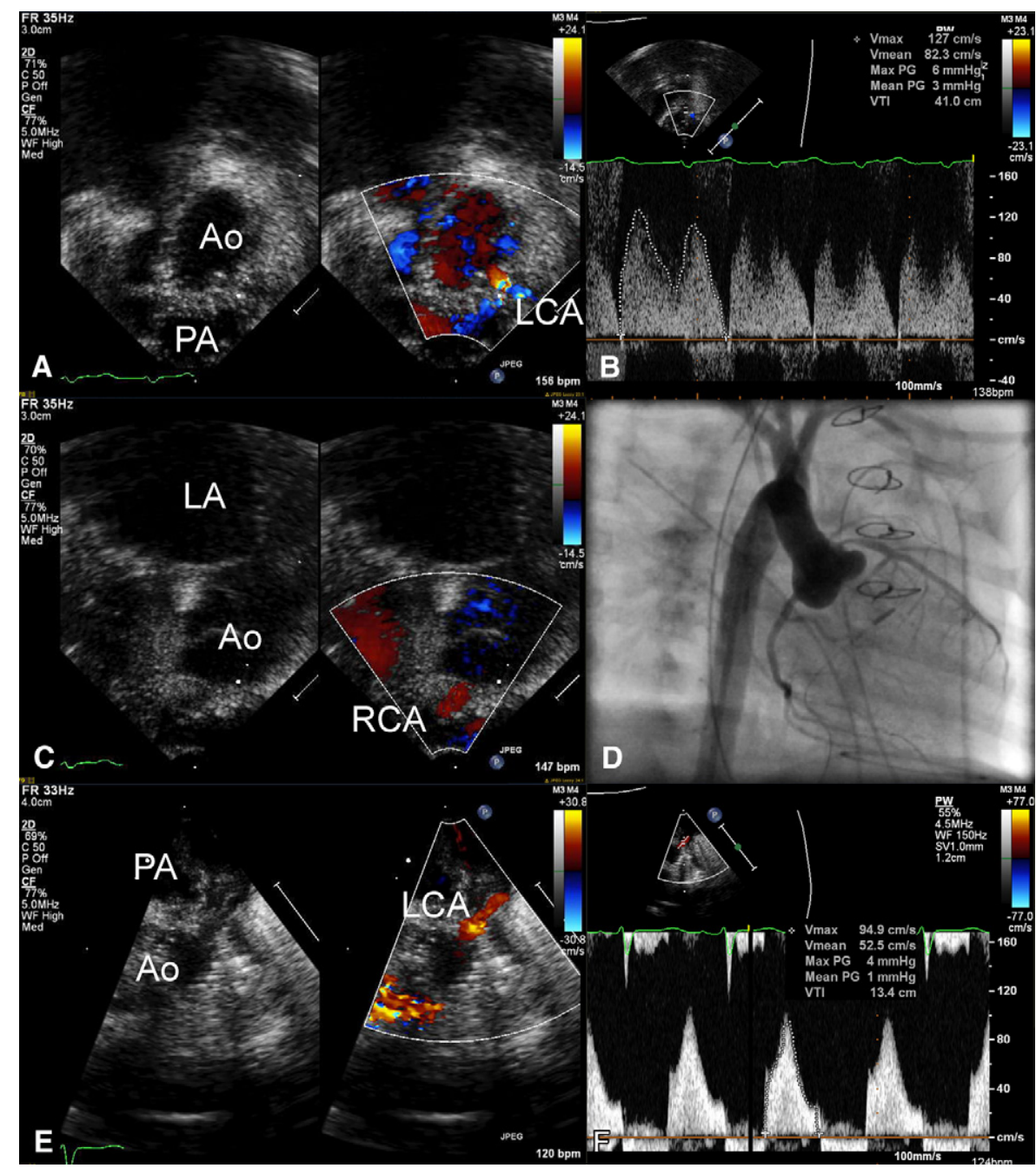

FIGURE 2. Neonate with transposition of the great arteries, ventricular septal defect, and usual coronary anatomy. As the patient was removed from bypass, TEE showed reduced ventricular function and laminar flow in the right coronary artery (C), but the left coronary artery could not be properly imaged. After surgical revision, EpE showed turbulent flow in the left coronary artery (A, B), which improved with further revision. At chest closure (day 5), there was severe ventricular dysfunction and electrocardiogram changes consistent with ischemia. Angiography (D) showed stenosis of left coronary artery leading to surgical revision. As the patient was removed from bypass, EpE showed laminar flow in the left coronary artery (E, F). Ao, Aorta; $L A$, left atrium; $L C A$, left coronary artery; $P A$, pulmonary artery; $R C A$, right pulmonary artery.

(Table 2). Both had a limited epicardial study after TEE probe extraction for hemodynamic instability. In 1 patient after an arterial switch operation, the left main coronary artery was reimplanted relatively high on the neoaortic root and was not well seen. It was subsequently proven to be stenotic by angiography the next day. Revision was performed after 4 days on extracorporeal membrane oxygenation. In the other patient, who underwent tetralogy of Fallot repair, the limited EpE was not used to image the distal branch pulmonary arteries. Suprasystemic right ventricle pressure necessitated fenestration of the ventricular septal defect patch. Severe bilateral pulmonary artery stenosis was diagnosed several months later and addressed by interventional catheterization.
Two patients in the TEE + EpE group required reintervention (Table 2). In 1 patient, there was severe ventricular dysfunction after mitral valve replacement. Epicardial imaging demonstrated laminar color flow in the distal left anterior descending and circumflex arteries, but the left main coronary vessel could not be visualized. Angiography 2 days later demonstrated obstruction of the left main coronary artery managed with stent implantation. The second patient had pulmonary atresia with ventricular septal defect and underwent repair with patch enlargement of the pulmonary arteries. During EpE, only the origins of the branch pulmonary arteries were visualized with only mild gradients obtained. Diffusely hypoplastic bilateral pulmonary arteries and severe left pulmonary artery 


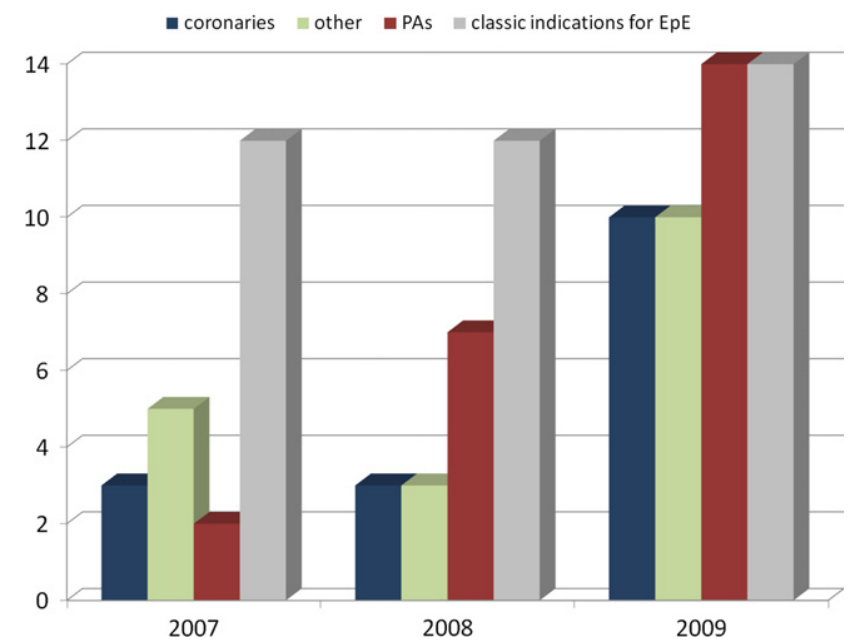

FIGURE 3. Use of EpE during the study period: 22 cases $(6 \%)$ in 2007; 25 cases $(6.3 \%)$ in $2008 ; 48$ cases $(10.9 \%)$ in $2009(P<.01)$. There was a significant increase in the use of EpE to obtain additional information. $P A$, Pulmonary artery.

stenosis were diagnosed 4 days later on angiography, and both pulmonary arteries were stented.

\section{DISCUSSION}

Historically, EpE was used in the operating room before and after cardiopulmonary bypass for the assessment of anatomic details and surgical results. ${ }^{6}$ During the introduction of biplane TEE, studies comparing the 2 techniques were conducted and TEE became the standard intraoperative imaging modality for congenital heart surgery. ${ }^{9,10}$ Its enormous beneficial clinical impact is well documented. ${ }^{7,8,12-18}$ TEE provides good visualization of the heart without interfering with the surgical field. It is the method of choice for imaging intracardiac structures and provides continuous information about cardiac filling and function. Nevertheless, there are limitations in imaging certain structures with TEE (ie, left pulmonary artery, aortic arch, left superior vena cava, collateral pulmonary vessels). ${ }^{11}$ These structures are better visualized by EpE.

The aim of this study was to examine the contemporary use of EpE. There has been an increase in surgical complexity along with an expectation of lower morbidity and mortality. Intraoperative imaging protocols have become more comprehensive, especially in complex cases. Understanding the anatomy of the repair, in conjunction with clinical and hemodynamic parameters, is essential to continuously improving outcomes.

At The Hospital for Sick Children, EpE is increasingly being used as an adjunct to TEE specifically to visualize cardiovascular structures that are difficult to image by TEE and are at risk of having residual abnormalities. Additional EpE was indicated most often for surgeries involving the pulmonary arteries (eg, pulmonary arterioplasty,
TABLE 1. Indications for surgical revisions

\begin{tabular}{|c|c|c|c|}
\hline \multirow{2}{*}{$\begin{array}{c}\text { Indications for } \\
\text { surgical revisions }\end{array}$} & \multirow{2}{*}{$\begin{array}{l}\text { TEE alone } \\
(\mathbf{n}=1109)\end{array}$} & \multicolumn{2}{|c|}{$\mathbf{E p E}(\mathbf{n}=95)$} \\
\hline & & EpE alone & $\mathbf{T E E}+\mathbf{E p E}$ \\
\hline Right ventricular outflow tract & 36 & 1 & - \\
\hline Atrioventricular valve & 29 & 1 & $1^{*}$ \\
\hline Coronary arteries & 12 & - & 3 \\
\hline $\begin{array}{l}\text { Venoatrial connections/baffles } \\
\text { (including pulmonary veins) }\end{array}$ & 10 & - & 1 \\
\hline Ventricular septal defect & 8 & - & - \\
\hline Pulmonary arteries & 7 & - & 5 \\
\hline Left ventricular outflow tract & 6 & - & - \\
\hline $\begin{array}{l}\text { Aortic arch/ Blalock- } \\
\text { Taussig shunt }\end{array}$ & 1 & - & 2 \\
\hline Total & $109(9.8 \%)$ & $2(5.3 \%)$ & $12(21.1 \%) \dagger$ \\
\hline
\end{tabular}

cavopulmonary anastomosis) and coronary arteries (eg, arterial switch, Ross procedure). In this regard, TEE and EpE seem to have complementary rather than exclusive applications to ensure comprehensive intraoperative imaging.

Residual branch pulmonary artery obstruction was the most common cause for intraoperative and postoperative reintervention, most often occurring in patients with a known history of obstruction. In the TEE alone group, it accounted for the majority of postoperative interventional procedures coinciding with lack of intraoperative echocardiographic imaging of these structures. This suggests the potential for vast improvement in the intraoperative identification of these lesions by EpE. There are significant limitations of TEE in imaging branch pulmonary arteries, particularly the left. Flexion of the TEE probe is usually needed when imaging pulmonary arteries, causing some distortion of the anatomy. Also, poor alignment often precludes correct assessment of pressure gradients. In contrast, EpE allows direct vessel visualization and adequate Doppler alignment.

TABLE 2. Missed residual lesions leading to early reintervention

\begin{tabular}{lccc}
\hline & TEE alone & \multicolumn{2}{c}{ EpE $(\mathbf{n}=\mathbf{9 5})$} \\
\cline { 3 - 4 } \multicolumn{1}{c}{ Missed residual lesions } & $(\mathbf{n}=\mathbf{1 1 0 9})$ & $\mathbf{E p E}$ alone & TEE $+\mathbf{E p E}$ \\
\hline Left pulmonary artery & $14^{*}$ & 1 & 1 \\
Right pulmonary artery & 6 & 1 & 1 \\
Glenn anastomosis & 6 & - & - \\
Coronary artery & $1^{*}$ & 1 & 1 \\
Main pulmonary artery & $1^{*}$ & - & - \\
Venoatrial connections & 1 & - & - \\
$\quad$ (including pulmonary veins) & & & - \\
Mitral valve & $1 *$ & - & - \\
Residual shunt & 2 & - & - \\
Total & 25 patients & 2 patients & 2 patients \\
& $(2.3 \%)$ & $(5.2 \%)$ & $(3.5 \%)$ \\
\hline
\end{tabular}

Several patients had mixed lesions (bilateral pulmonary arteries or Glenn and pulmonary artery). *Four patients in whom the residual lesion was imaged but the result was misinterpreted. 
In specific cases, residual stenosis may be accepted by the surgical team, but the knowledge of their existence may influence postoperative management. Our intraoperative protocol has evolved over the years as EpE imaging use has increased. If the TEE does not visualize the branch pulmonary arteries or there is elevation in right ventricular pressure by echocardiography or direct pressure, pulmonary artery pressures are obtained. If there is a pressure gradient, $\mathrm{EpE}$ is generally performed to visualize the site of obstruction.

Another category of patients who would benefit from additional epicardial imaging are those with single-ventricle physiology, in whom imaging the cavopulmonary anastomosis is often challenging. EpE allows good visualization of the entire cavopulmonary circuit, with particular utility in the case of bilateral superior caval veins.

There was a significant increase during the study period in the use of EpE for coronary artery imaging after reimplantation (arterial switch operation, Ross procedure, and anomalous left coronary artery from pulmonary artery repair), recognizing that coronary abnormalities are a major cause of morbidity and mortality in this population. ${ }^{19}$ Our experience shows that EpE can be useful in imaging coronary arteries when TEE views are limited.

The comparison of patient outcomes (intraoperative revision, postoperative morbidity, and reintervention) between the period in which our institution was using TEE alone and the current TEE + EpE protocol was beyond the scope of this review. The increased use of EpE resulted in a higher detection rate of residual lesions and increased intraoperative revisions. Theoretically, this may improve outcomes, specifically postoperative course and need for reintervention. Overall, the incidence of residual lesions in all patients is relatively low, and the indication for EpE must factor in different elements, including the type of procedure, preoperative risk factors, TEE findings, and postoperative hemodynamics.

The echocardiographic "misses" that occurred, even with the addition of EpE, have led to a modification of our intraoperative imaging protocol for certain cardiac conditions. In the learning curve of the hybrid procedure for single-ventricle management, intraoperative or exit angiography of the cavopulmonary anastomosis is now performed routinely after second stage palliation. Particularly after the bilateral bidirectional Glenn procedure, the left pulmonary artery may be distorted and obstructed. ${ }^{20}$ Exit angiography is also performed after unifocalization of aortopulmonary collateral vessels, because imaging is superior to TEE or EpE.

\section{Limitations}

There are limitations related to retrospective data collection and analysis. The indications for EpE were not always available but were implied on the basis of the structures imaged. Surgical outcome was not evaluated beyond 1 year postoperatively. In particular, we do not compare postoperative reintervention, morbidity, and mortality rates between TEE alone and TEE + EpE. We acknowledge there is no gold standard for determining whether an identified residual lesion warrants revision intraoperatively or could be deferred. This decision was made by the individual surgeon on the basis of the comprehensive clinical scenario.

\section{CONCLUSIONS}

Use of epicardial imaging in the operating room may further increase the identification of postoperative residual lesions, mainly related to branch pulmonary arteries, cavopulmonary anastomosis, and coronary arteries. When these structures are not adequately visualized by TEE, adjunct EpE can help identify residual problems, guiding postoperative management and potentially decreasing reintervention rates.

\section{References}

1. Gersony WM. Long-term follow-up of operated congenital heart disease. Cardiol Clin. 1989;7:915-23.

2. Warnes CA. The adult with congenital heart disease: born to be bad? J Am Coll Cardiol. 2005;46:1-8.

3. Cyran SE, Kimball TR, Meyer RA, et al. Efficacy of intraoperative transesophageal echocardiography in children with congenital heart disease. Am J Cardiol. 1989;63:594-8.

4. Gussenhoven EJ, van Herwerden LA, Roelandt J, Ligtvoet KM, Bos E, Witsenburg M. Intraoperative two-dimensional echocardiography in congenital heart disease. J Am Coll Cardiol. 1987;9:565-72.

5. Stumper OF, Elzenga NJ, Hess J, Sutherland GR. Transesophageal echocardiography in children with congenital heart disease: an initial experience. J Am Coll Cardiol. 1990;16:433-41.

6. Ungerleider RM, Greeley WJ, Sheikh KH, et al. Routine use of intraoperative epicardial echocardiography and Doppler color flow imaging to guide and evaluate repair of congenital heart lesions. A prospective study. J Thorac Cardiovasc Surg. 1990;100:297-309.

7. Ungerleider RM, Kisslo JA, Greeley WJ, et al. Intraoperative echocardiography during congenital heart operations: experience from 1,000 cases. Ann Thorac Surg. 1995;60:S539-42.

8. Randolph GR, Hagler DJ, Connolly HM, et al. Intraoperative transesophageal echocardiography during surgery for congenital heart defects. J Thorac Cardiovasc Surg. 2002;124:1176-82.

9. Muhiudeen IA, Roberson DA, Silverman NH, Haas G, Turley K, Cahalan MK. Intraoperative echocardiography in infants and children with congenital cardiac shunt lesions: transesophageal versus epicardial echocardiography. J Am Coll Cardiol. 1990;16:1687-95.

10. Stumper O, Kaulitz R, Sreeram N, et al. Intraoperative transesophageal versus epicardial ultrasound in surgery for congenital heart disease. J Am Soc Echocardiogr. 1990;3:392-401.

11. Ayres NA, Miller-Hance W, Fyfe DA, et al. Indications and guidelines for performance of transesophageal echocardiography in the patient with pediatric acquired or congenital heart disease: report from the task force of the Pediatric Council of the American Society of Echocardiography. J Am Soc Echocardiogr. 2005; $18: 91-8$

12. Hsu YH, Santulli T Jr, Wong AL, Drinkwater D, Laks H, Williams RG. Impact of intraoperative echocardiography on surgical management of congenital heart disease. Am J Cardiol. 1991;67:1279-83.

13. Stumper O, Kaulitz R, Elzenga NJ, et al. The value of transesophageal echocardiography in children with congenital heart disease. J Am Soc Echocardiogr. 1991;4:164-76.

14. Stevenson JG, Sorensen GK, Gartman DM, Hall DG, Rittenhouse EA. Transesophageal echocardiography during repair of congenital cardiac defects: identification of residual problems necessitating reoperation. J Am Soc Echocardiogr. 1993;6:356-65. 
15. Bengur AR, Li JS, Herlong JR, Jaggers J, Sanders SP, Ungerleider RM. Intraoperative transesophageal echocardiography in congenital heart disease. Semin Thorac Cardiovasc Surg. 1998;10:255-64.

16. Muhiudeen Russell IA, Miller-Hance WC, Silverman NH. Intraoperative transesophageal echocardiography for pediatric patients with congenital heart disease. Anesth Analg. 1998;87:1058-76.

17. Rosenfeld HM, Gentles TL, Wernovsky G, et al. Utility of intraoperative transesophageal echocardiography in the assessment of residual cardiac defects. Pediatr Cardiol. 1998;19:346-51.
18. Cohen GA, Stevenson JG. Intraoperative echocardiography for atrioventricular canal: decision-making for surgeons. Semin Thorac Cardiovasc Surg Pediatr Card Surg Annu. 2007;47-50.

19. Wong D, Golding F, Hess L, et al. Intraoperative coronary artery pulse Doppler patterns in patients with complete transposition of the great arteries undergoing the arterial switch operation. Am Heart J. 2008;156:466-72.

20. Iyer GK, Van Arsdell GS, Dicke FP, McCrindle BW, Coles JG, Williams WG. Are bilateral superior vena cavae a risk factor for single ventricle palliation? Ann Thorac Surg. 2000;70:711-6.

Access to The Journal of Thoracic and Cardiovascular Surgery Online is reserved for print subscribers!

Full-text access to The Journal of Thoracic and Cardiovascular Surgery Online is available for all print subscribers. To activate your individual online subscription, please visit The Journal of Thoracic and Cardiovascular Surgery Online, point your browser to http://www.mosby.com/jtcvs, follow the prompts to activate your online access, and follow the instructions. To activate your account, you will need your subscriber account number, which you can find on your mailing label (note: the number of digits in your subscriber account number varies from 6 to 10). See the example below in which the subscriber account number has been circled:

\section{Sample mailing label}

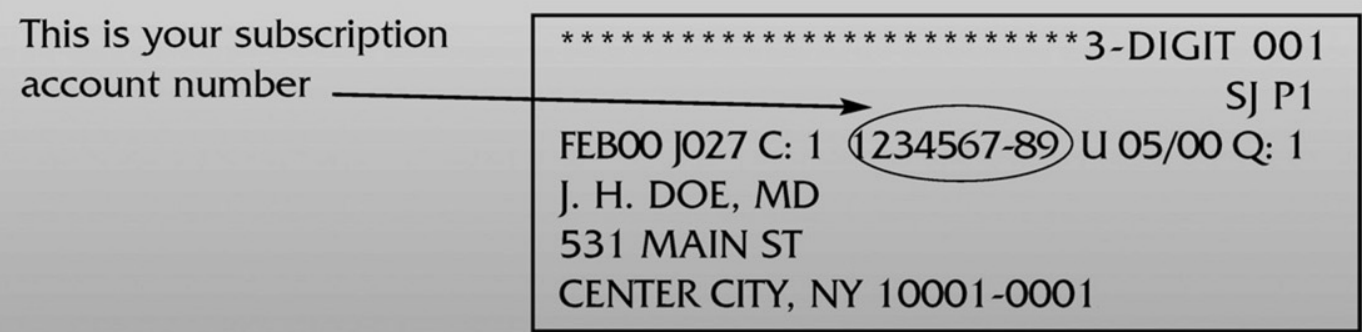

Personal subscriptions to The Journal of Thoracic and Cardiovascular Surgery Online are for individual use only and may not be transferred. Use of The Journal of Thoracic and Cardiovascular Surgery Online is subject to agreement to the terms and conditions as indicated online. 
TABLE E1. Cardiac diagnostic groups

\begin{tabular}{|c|c|c|}
\hline & IOE & $\mathbf{T E E}+\mathbf{E p E}$ \\
\hline Shunt (ASD/VSD/AVSD) & 295 & - \\
\hline RVOT (TF/CAT/RV-PA) & 269 & $18(6.7 \%)$ \\
\hline Glenn/Fontan & 202 & $9(4.5 \%)$ \\
\hline Arterial switch operation & 90 & $6(6.7 \%)$ \\
\hline LVOT/ascending aorta (including Ross) & 86 & $6(7 \%)$ \\
\hline Atrioventricular valves & 62 & $2(3.2 \%)$ \\
\hline $\begin{array}{l}\text { Venoatrial connections/baffles } \\
\text { (including pulmonary veins) }\end{array}$ & 60 & $4(6.7 \%)$ \\
\hline Norwood & 25 & $6(24 \%)$ \\
\hline Coronary & 19 & - \\
\hline Other & 96 & $6(6.2 \%)$ \\
\hline Total & 1204 & $57(4.7 \%)$ \\
\hline \multicolumn{3}{|c|}{$\begin{array}{l}\text { Percentages represent the use of EpE for a specific diagnostic group. } A S D \text {, Atrial sep- } \\
\text { tal defect (only primum and sinus venosus ASD included); } A V S D \text {, atrioventricular } \\
\text { septal defect; } C A T \text {, common arterial trunk; } I O E \text {, intraoperative echocardiography; } \\
L V O T \text {, left ventricular outflow tract; } R V-P A \text {, right ventricle to pulmonary artery con- } \\
\text { duit placement/replacement; } T F \text {, tetralogy of Fallot and variants; VSD, ventricular } \\
\text { septal defect. }\end{array}$} \\
\hline
\end{tabular}

